Igor Nenadic • Christian Gaser • Hans-Peter Volz • Thomas Rammsayer · Frank Häger · Heinrich Sauer

\title{
Processing of temporal information and the basal ganglia: new evidence from fMRI
}

Received: 3 March 2000 / Accepted: 11 October 2001 / Published online: 27 November 2002

(C) Springer-Verlag 2002

\begin{abstract}
Temporal information processing is a fundamental brain function, which might include central timekeeping mechanisms independent of sensory modality. Psychopharmacological and patient studies suggest a crucial role of the basal ganglia in time estimation. In this study, functional magnetic resonance imaging (fMRI) was applied in 15 healthy right-handed male subjects performing an auditory time estimation task (duration discrimination of tone pairs in the range of 1,000 $1,400 \mathrm{~ms}$ ) and frequency discriminations (tone pairs differing in pitch, around $1,000 \mathrm{~Hz}$ ) as an active control task. Task difficulty was constantly modulated by an adaptive algorithm (weighted up-down method) reacting on individual performance. Time estimation (vs rest condition) elicited a distinct pattern of cerebral activity, including the right medial and both left and right dorsolateral prefrontal cortices (DLPFC), thalamus, basal ganglia (caudate nucleus and putamen), left anterior cingulate cortex, and superior temporal auditory areas. Most activations showed lateralisation to the right hemisphere and were similar in the frequency discrimination task. Comparing time and frequency tasks, we isolated activation in the right putamen restricted to time estimation only. This result supports the notion of central processing of temporal information associated with basal ganglia activity. Temporal information processing in the brain might thus be a distributed process of interaction between modality-dependent sensory cortical function, the putamen (with a timing-specific function), and additional prefrontal cortical systems related to attention and memory. Further investigations are needed to delin-
\end{abstract}

I. Nenadic $(\bowtie) \cdot$ C. Gaser · H.-P. Volz · F. Häger · H. Sauer Department of Psychiatry, Friedrich-Schiller-University of Jena, Philosophenweg 3, 07740 Jena, Germany

e-mail: igor.nenadic@uni-jena.de

Tel.: +49-3641-935417/935805

Fax: +49-3641-935280

T. Rammsayer

Georg-Elias-Müller-Institute for Psychology,

Georg-August-University of Göttingen, Gosslerstrasse 14,

37073 Göttingen, Germany eate the differential contributions of the striatum and other areas to timing.

Keywords Time perception - Internal clock - Putamen • Basal ganglia - Cortex - Prefrontal cortex - Functional magnetic resonance imaging

\section{Introduction}

Temporal information processing is a fundamental aspect of central nervous system function. Both perception and action are embedded in the framework of a precise temporal coordination of information processing and encoding. The computations involved in such processes are manifold, as temporal information processing might refer to a variety of functions, for example processing of the duration of stimuli, their temporal order, or retrieving a sequence of events from memory. Many neurophysiological approaches to time and temporal information processing have been related to the processing of information in time, timeliness and temporal order (see Fuster 1995). However, this is not the case for timing in the sense of processing the temporal information of a stimulus itself, i.e. its duration. This function is usually assessed in paradigms of estimation, discrimination or reproduction of intervals with different length (Fraisse 1957, 1963). In recent decades, a rich fundament of psychophysics related to timing in animals and humans has been established (Gibbon and Allan 1984; Allan 1979), providing a theoretical and mathematical framework. However, it is only recently that a "neurobiology of temporal cognition" has emerged (Gibbon et al. 1997; see also Ivry 1996), in the sense of an integrative theory of the processing of temporal information per se. This approach relates aspects from time psychology to the question of underlying neural "mechanisms" and brain processes (Hazeltine et al. 1997). The concept of an "internal clock" of the brain refers to a hypothetical unit (or multiple units) in the central nervous system providing the modalityindependent timekeeper function necessary for temporal 
information processing in the range of milliseconds to seconds.

Neuropsychological models of timing (e.g. Gibbon et al. 1997; Nichelli 1993) have been established, which try to explain processes during tasks such as duration discrimination. The core of these models is often thought to be a central "clock" unit, and has been suggested to be represented as an oscillating pacemaker module. Together with memory and decision-making components these models explain the established mathematical and psychophysical properties of, for example, duration comparison. Hence, one might distinguish "modules" or substrates of primary or "central" timing (i.e. the "internal clock" in a narrow sense) and "secondary" components promoting additional functions such as memory, attention and decision-making.

The "secondary modules" underlying performance of time discrimination might be effectively the same for a similar non-temporal discrimination task. Characterising timing deficits in patients therefore includes testing on similar tasks to differentiate between disorders of the central timing component and less specific general functions. Cortical lesions, for example, can have profound effects on timing performance, depending on extent and location. This has been demonstrated in several studies (with different testing procedures) for prefrontal, but also parietal areas (Mangels et al. 1998; Harrington et al. 1998b; Damasceno 1996). However, performance on control tasks (e.g. frequency or intensity discrimination) is also affected, suggesting less specific functions for these regions. A similar dissociation also seems to be apparent in differential performance of amnestic and Alzheimer's disease patients (Nichelli et al. 1993), where only the latter group revealed an involvement of both central clock and memory/decision processes.

For central or "explicit" timing functions, the basal ganglia and the cerebellum have been implied. A major line of evidence for the basal ganglia arises from studies on Parkinson's disease (PD), which serves as a model of decreased dopaminergic function in the basal ganglia, and particularly depleting input to the putamen (Brooks et al. 1990). After initial negative results (Ivry and Keele 1989), several studies have shown and confirmed an impairment on different timing tasks in PD (e.g. Artieda et al. 1992; Pastor et al. 1992; Harrington et al. 1998a), which is especially prominent when patients are taken off dopaminergic medication (Malapani et al. 1998). This deficit is not restricted to motor timing, but is also evident in perception, for example in discrimination of temporal intervals (Hellström et al. 1997; Rammsayer and Classen 1997). Additionally, a wealth of data from pharmacopsychological studies point to the role of dopamine (DA) as the major transmitter for temporal information processing (for overviews, see Meck 1996; Rammsayer 1997a). Differential manipulation with DA antagonists in humans has suggested in particular the nigrostriatal dopaminergic system as a potential substrate of central clock processes (Rammsayer 1997b).
Direct approaches of detecting timing-related brain activity in normals, such as neuroimaging with positron emission tomography (PET) or functional magnetic resonance imaging (fMRI), have remained comparably sparse, especially for perceptual timing operations. While some related investigations did not find "timing-specific" activations (Maquet et al. 1996), others have given support to the cerebellum (Jüptner et al. 1995). The basal ganglia and the cerebellum have also been implicated in studies of motor timing (Rao et al. 1997; Penhune et al. 1998), but it is not clear to what degree perceptual and motor timing show overlap in functional neurobiological organisation.

The aim of our study was to investigate basal ganglia activity using fMRI during a perceptual timing task. We hypothesised that activity in areas like the dorsolateral prefrontal cortex (DLPFC) and the anterior cingulate cortex would occur similarly in active control tasks, reflecting memory and attentional demands of the tasks, whereas differences in activations would be evident in a certain part of the basal ganglia, supporting the notion of "timing-specific" activations.

\section{Materials and methods}

\section{Subjects}

In this study, a total of 15 healthy male adults were included [mean age 25.3 years, standard deviation (SD) 3.6, range 21-31 years]. From the initially recruited 18 volunteers two had to be excluded because of head movements during scanning (exceeding the criteria explained below) and one due to technical malfunction of the response device. All subjects were strongly right handed as assessed by the Edinburgh handedness scale (Oldfield 1971), and no professional musicians were included. The participants were thoroughly interviewed for any psychiatric or neurological history, as well as for complications during birth or major abnormalities in childhood development. Further exclusion criteria were: major neurological or psychiatric disorders in first and second degree relatives, internal medical conditions, current medication possibly affecting mental state or performance, and current or previous substance abuse or dependence. All subjects had at least 10 years (usually more than 12 years) of school education. After an explanation of the study, all volunteers gave written informed consent. The study was approved by the ethics review committee of the Friedrich-Schiller University of Jena.

\section{Behavioural tasks}

The paradigm included two tasks: time estimation and frequency discrimination (i.e. pitch discrimination) of pairs of tones. The presentation of auditory stimuli and recording of the subject responses was controlled by a standard personal computer. Auditory stimuli were generated by a Soundblaster soundcard and presented through an MR-compatible pneumatic headphone system, with a constant amplitude well above scanner noise. Within each trial, subjects were presented two auditory intervals (1,000-Hz sine waves), a constant standard interval and a variable comparison interval. The duration of the standard interval was $1,000 \mathrm{~ms}$. The initial value of the comparison interval was $1,400 \mathrm{~ms}$. For quantification of performance on time estimation, the weighted up-down method, an adaptive psychophysical procedure, was used (Kaernbach 1991; Rammsayer 1992). "Adaptive" means that the comparison interval varied in duration from trial to trial depending 
on the subject's previous response. Correct responding resulted in a decrease of the duration of the comparison interval and incorrect responses made the task easier by increasing the duration of the comparison interval. In the present study, this adaptive procedure was used to determine the $75 \%$ difference threshold as an indicator of performance on time estimation. This threshold represents the mean difference in duration between the standard and the comparison interval required to produce $75 \%$ correct responding, with better performance being indicated by smaller threshold values. Individual threshold values were computed based on midrun estimates (Wetherill et al. 1966). The duration of the comparison interval changed with a basic step size of $S=400 \mathrm{~ms}$ for trials $1-6$, resulting in a downward step of $S_{\text {down }}=100 \mathrm{~ms}$ and an upward step of $S_{\text {up }}=300 \mathrm{~ms}$, and $S=100 \mathrm{~ms}$ for the following trials, resulting in a downward step of $S_{\text {down }}=25 \mathrm{~ms}$ and an upward step of $S_{\text {up }}=75 \mathrm{~ms}$, respectively. Order of presentation for the standard interval and the comparison interval was randomised and balanced, with each order being presented in $50 \%$ of the trials. The two intervals were presented with an interstimulus interval (ISI) of $900 \mathrm{~ms}$. The subject's task was to decide which of the two intervals was longer and to indicate his decision by pressing one of two designated pneumatic response buttons. Before the fMRI experiment, subjects were given 12 practice trials and a baseline measurement consisting of 36 trials. Under the fMRI condition, three blocks (3:06 min each) of time estimation were performed by each subject, each starting with the initial standard values and allowing the algorithm to reliably approach and hold the designated threshold.

The frequency discrimination task was similar to time estimation except that the two auditory stimuli presented within each trial differed in frequency only and subjects indicated the higher tone. The weighted up-down method was started with the standard interval of $1,000 \mathrm{~Hz}$ and the initial comparison interval of $1,015 \mathrm{~Hz}$, and a basic step size of $S=24 \mathrm{~Hz}$ for trials 1-6 (resulting in $S_{\text {down }}=6 \mathrm{~Hz}$ and $S_{\text {up }}=18 \mathrm{~Hz}$ ) and $S=8 \mathrm{~Hz}$ for the following trials $\left(S_{\text {down }}=2 \mathrm{~Hz}\right.$ and $S_{\text {up }}=6 \mathrm{~Hz}$, respectively). In the frequency discrimination task, the duration of all stimuli was $1,000 \mathrm{~ms}$. Otherwise, the procedure was the same as described for the time estimation task.

\section{Imaging procedure}

Functional imaging was performed on a 1.5-Tesla MR scanner (Philips Gyroscan ACSII, Philips, Netherlands). An oblique stack of four slices was positioned on the basis of the AC-PC line (between the anterior and the posterior commissure) and an additional perpendicular line on the anterior commissure (for details of the procedure, see Volz et al. 1997; Häger et al. 1998). This stack orientation was selected to cover the regions of interest: particularly the basal ganglia, but also the DLPFC, thalamus, anterior cingulate cortex, and superior temporal cortex. For anatomical correlation, T1-weighted images were acquired [spin echo sequence, $\mathrm{TR}=300 \mathrm{~ms}$, $\mathrm{TE}=15 \mathrm{~ms}, \alpha=90^{\circ}$, matrix of $256 \times 256$, in-plane field of view (FOV) $230 \mathrm{~mm}$, and a resulting voxel size of $0.9 \times 0.9 \times 10 \mathrm{~mm}]$. The fMRI procedure was performed with a T2*weighted fast field echo (FFE) sequence $(\mathrm{TR}=100 \mathrm{~ms}, \mathrm{TE}=50 \mathrm{~ms}$, $\alpha=15^{\circ}$, in-plane matrix $128 \times 128$, in-plane FOV $230 \mathrm{~mm}$, slice thickness $10 \mathrm{~mm}$, resulting voxel size of $1.8 \times 1.8 \times 10 \mathrm{~mm}$ ). Subsampling of k-space with zero-filling was applied for this FFE sequence, resulting in a duration of $31 \mathrm{~s}$ for each volume set. Subjects' heads were positioned in a holding device modelled for the headphone capsules, and pads were used to further restrict potential head movement.

A blocked design with three conditions was applied: rest (R), time estimation (T), and frequency discrimination (F). During the resting state, subjects had to keep their eyes closed, received no auditory task stimulation, and were not to perform any motor actions. During $\mathrm{T}$ and $\mathrm{F}$ conditions, the subjects performed the task as described above. The fMRI experiment started with a first image (with subjects resting), allowing the MR signal to reach equilibrium and therefore not being further processed in the following analysis.
The scheme of the blocked experiment was an alternating row of 1:33 min (three images) blocks of rest (R) and 3:06 min (six images) blocks of an active condition (either T or F). The order of active conditions in this scheme was counterbalanced. This means that subjects either had a rest-time-rest-frequency-rest-... or restfrequency-rest-time-rest-... scheme. Over the entire fMRI experiment, a total of three blocks of $\mathrm{T}$ and three blocks of $\mathrm{F}$ (3×3:06 min), as well as six blocks of $\mathrm{R}$ (each 1:33 min) was obtained. The total duration of each condition ( $\mathrm{T}, \mathrm{F}$, or $\mathrm{R})$ in one fMRI experiment was therefore identical (9:18 min each). During each of the active blocks ( $\mathrm{T}$ or $\mathrm{F}$ ), the adaptive algorithm was initiated with the mentioned starting values for the $\mathrm{T}$ and $\mathrm{F}$ tasks. Use of either left or right hand for responses was also counterbalanced over subjects (still all subjects were strongly right-handed).

\section{Image analysis}

The fMRI data set was analysed using SPM96 (Statistical Parametric Mapping) from the Wellcome Department of Cognitive Neurology, London (Friston et al. 1995), and the random effects tool kit for SPM96 (Holmes and Friston 1998). First, the scans were realigned to the first scan, which was checked for the absence of motion artefacts. Three parameters (rotation, translation $x$ and $y$ ) were estimated using a least squares approach (Frackowiak et al. 1997). Subjects whose images exhibited a translation of $2 \mathrm{~mm}$ or more, or a rotation of $2^{\circ}$ or more, were not included in the study. Two of the initial 18 subjects were therefore rejected. In order to spatially normalise the images, thus facilitating intersubject averaging, the data from different subjects had to be transformed into a standard anatomical space. The parameters for the spatial normalisation were estimated in two steps. First, the images were normalised to a predefined four-slice template using a twodimensional affine transformation. In the second step, this fourslice template was normalised to the standard template in the SPM package performing a three-dimensional affine transformation, thus allowing the use of the Talairach space of the SPM template. To assess appropriate population inference, we used the random effects tool kit for SPM96 (Holmes and Friston 1998). Data were collapsed over replications to give a single summary image representative of each condition for each subject. This collapsing was performed by a weighted mean of adjusted data effected via the General Linear Model. Images were trend-corrected by fitting a cubic polynomial function to the global mean values of the time series and using this fit for proportional scaling. To facilitate intersubject averaging and to conform to the Gaussian field theory, data were smoothed with an isotropic Gaussian kernel with full width at half maximum (FWHM) of $6 \mathrm{~mm}$. Statistical analysis was performed as a multisubject design in SPM96. Contrasts were computed for T vs $\mathrm{R}$ and $\mathrm{F}$ vs $\mathrm{R}$ conditions. A differential contrast of $\mathrm{T}$ vs $\mathrm{F}$ was analysed at a lower, hypothesis-led threshold level to show potential timing-related basal ganglia activations. In this contrast, we also analysed parameter estimates for selected clusters. These parameter estimates are the results of the statistical assessment in the general linear model applied. The linear equations set up for each voxel contain an error term, as well as a term for each condition, which again consists of a coefficient and the condition variable (to be set in different contrasts). The parameter estimates describe the calculated coefficients in this voxel and approximate the effects during $\mathrm{R}, \mathrm{T}$, and $\mathrm{F}$ conditions (see also Friston et al. 1995).

\section{Results}

The results of the behavioural data are given in Table 1. As demonstrated, performance on the tasks during scanning was not impaired, showing that the tasks could be reliably performed by subjects despite scanner noise. To exclude time or learning effects during the scanner 
Table 1 Task performance as indicated by the $75 \%$ difference thresholds. Mean and standard deviations (SD) are presented for the previous baseline testing and for the fMRI trials. In the latter case, both overall performance is given (T-mean) as well as in the three separate blocks performed for each task (T-1 to T-3)

Table 2 Activations during time estimation compared to rest

Fig. 1A, B Activations during time estimation compared to rest, same thresholds as given in Table 1. Significant voxels less than $30 \mathrm{~mm}$ from the cortex surfaces are superimposed on the template surface (lateral and medial views)

\begin{tabular}{lll}
\hline & Time estimation $(\mathrm{ms})$ & Frequency discrimination $(\mathrm{Hz})$ \\
\hline Baseline testing & $149 \pm 57$ & $5.3 \pm 2.4$ \\
T-mean & $124 \pm 27$ & $4.6 \pm 0.9$ \\
T-1 & $131 \pm 62$ & $4.9 \pm 1.4$ \\
T-2 & $118 \pm 46$ & $4.2 \pm 1.2$ \\
T-3 & $115 \pm 47$ & $4.9 \pm 1.5$ \\
\hline
\end{tabular}

\begin{tabular}{lll}
\hline$x, y, z$ & $z$ score & Region (approx. Brodmann's area) \\
\hline $38,42,26$ & 4.84 & Right middle frontal gyrus (DLPFC, BA 46) \\
$54,16,14$ & 4.80 & Right inferior frontal gyrus (44/45) \\
$10,28,36$ & 5.55 & Right medial frontal cortex (32) \\
$36,30,2$ & 5.10 & Right inferior frontal cortex (45/47)/right anterior insula \\
$-34,24,2$ & 4.14 & Left insula \\
$-12,34,26$ & 4.22 & Left anterior cingulate/cingulate sulcus (24) \\
$-62,-12,10$ & 4.28 & Left superior/transverse temporal gyrus (41/42) \\
$66,-8,12$ & 4.08 & Right superior/transverse temporal gyrus $(41 / 42)$ \\
$66,-28,4$ & 5.64 & Right superior and middle temporal gyrus $(22 / 21)$ \\
$12,2,16$ & 4.36 & Right caudate nucleus \\
$6,-8,10$ & 4.52 & Right thalamus (anterior/mediodorsal nuclei) \\
$-32,-2,-2$ & 3.94 & Left putamen \\
$28,16,-6$ & 3.13 & Right putamen \\
\hline
\end{tabular}

Local max. voxels, height $P=0.001$, uncorrected, extent threshold $k=60$ voxels; note that Brodmann's areas, as given in brackets, only represent an approximation
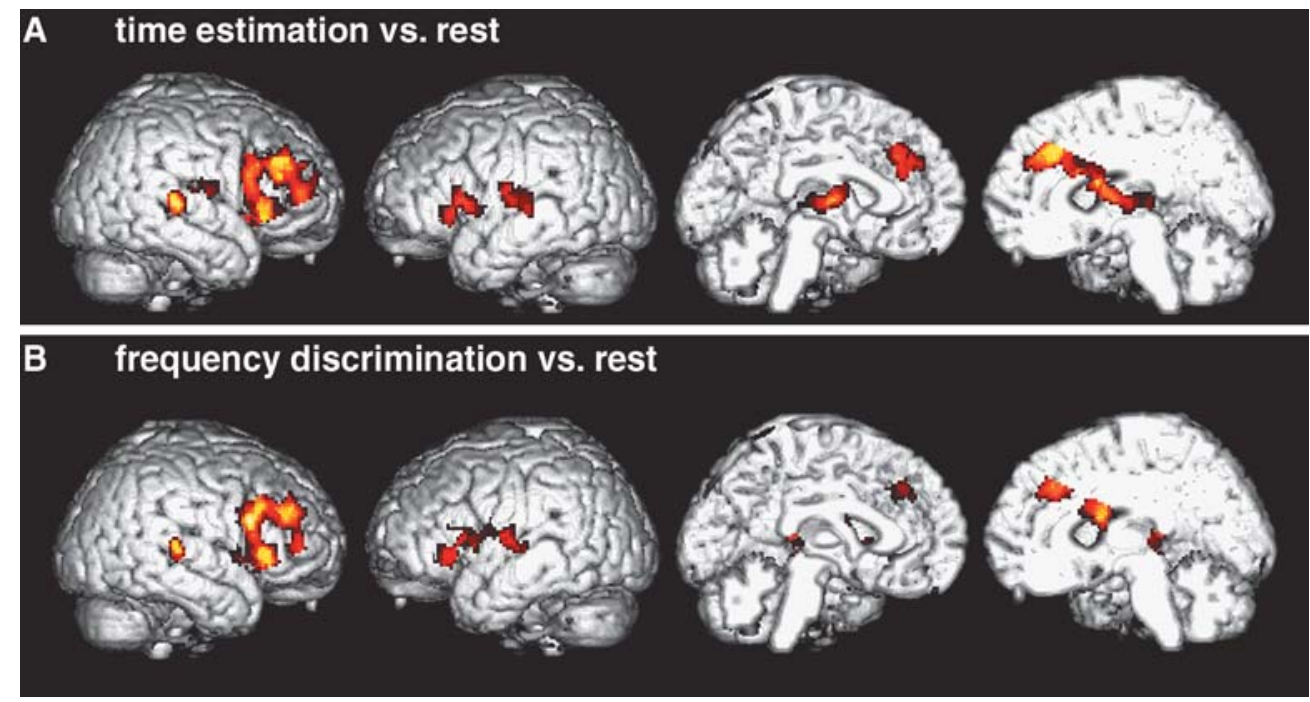

session, we analysed performance data of single blocks (T1, T2, T3) using repeated measures analysis of variance (ANOVA). There was no significant effect of time for either time estimation $\left(F_{(2,28)}=0.39, P=0.68\right)$ or frequency discrimination $\left(F_{(2,28)}=1.49, P=0.24\right)$.

The analysis of the fMRI data consists of separate testing of different contrast. Resulting voxel-wise $z$ statistics are shown in different formats. Table 2 shows activated regions contrasting time estimation $(\mathrm{T})$ versus rest $(\mathrm{R})$ conditions. Besides bilateral auditory cortex activation, prefrontal cortical signal increases occurred in the middle and inferior frontal gyri of the right hemi- sphere and the left inferior frontal gyrus. Anterior cingulate cortex (cingulate sulcus), caudate nucleus, and putamen activations were also encountered as displayed. The pattern of activity of frequency discrimination $(\mathrm{F})$ versus rest $(\mathrm{R})$ was very similar to the one obtained during time estimation. Figure 1 gives views of the lateral and medial surfaces of the brain with activated cortical clusters rendered on the surface.

Based on our hypothesis for the basal ganglia, we computed the difference between time estimation and frequency discrimination ( $\mathrm{T}$ vs $\mathrm{F}$ contrast) on a level of $P<0.05$. The only cluster in the region, for which the 

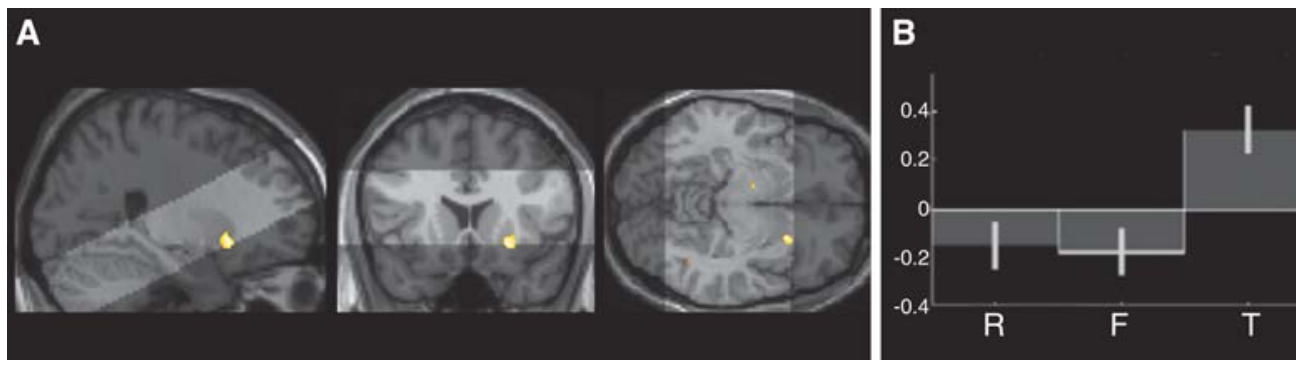

Fig. 2A, B Differences of activations between time estimation $(T)$ and frequency discrimination $(F)$ in the right putamen $(26,18,-8)$ at $P<0.05$. The cluster is superimposed on three orthogonal sections of the template image. Note that the studied brain volume/stack of

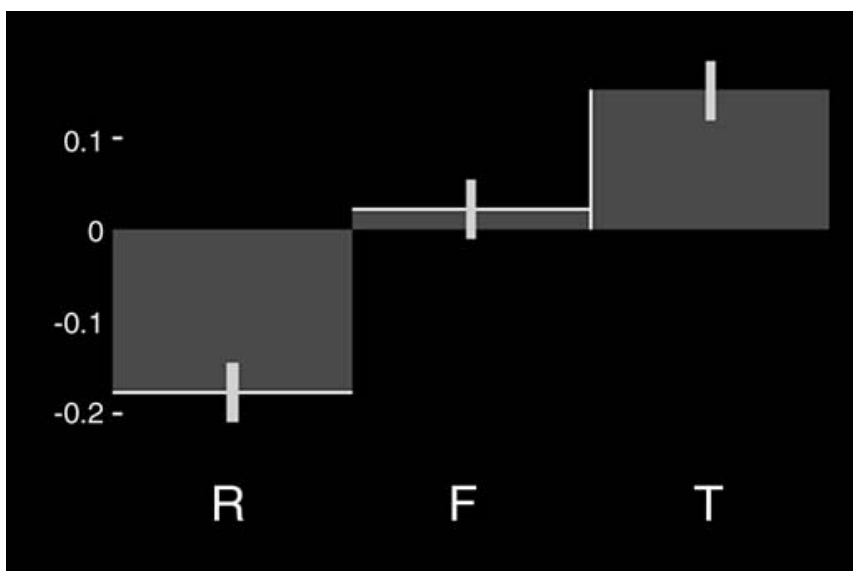

Fig. 3 Parameter estimates for right ventrolateral prefrontal/insular cortical cluster $(P<0.05)$

anatomical hypothesis was defined, was encountered in the right putamen. This result is displayed in Fig. 2, showing the activated cluster in coronal, sagittal, and transversal sections, and bar diagrams for parameter estimates in the local maximum voxel. These parameter estimates illustrate the activation profile, demonstrating the specific rise in signal intensity during the blocks of the time estimation condition (T). Beyond this putamen cluster, we found voxels which also showed a significant result in the $\mathrm{T}$ vs $\mathrm{F}$ contrast, but were located outside the specified region, for which the hypothesis was defined, and thus can only be described as trend results. In fact, most clusters in this analysis actually showed relative signal decrease during $\mathrm{F}$, rather than timing-related activation, and therefore voxels with such activation profiles have to be rejected as not being timing specific.

However, we located a cluster of significant voxels in a region at the junction of the right ventrolateral prefrontal cortex and insular cortex, which showed a distinct activation profile. As seen in Fig. 3, these voxels showed a (significant) rise in signal intensity during time estimation (vs rest), but to a lesser extent also during frequency discrimination (vs rest). Although this region was not included in the initial hypothesis and therefore has to be treated as a trend finding, it is shown to be due slices is brightened. The diagram shows the parameter estimates for the three conditions: rest $(\mathrm{R})$, frequency discrimination $(\mathrm{F})$, and time estimation (T)

to a potential role of this anatomical structure. Resolution was not sufficient to definitely resolve whether this cluster was located more in the ventrolateral or insular cortex, so interpretations will be restricted. Note that elevation of signal intensity (versus rest) does not exclusively occur during the timing, but to a lesser extent also during the frequency condition.

\section{Discussion}

In this study, we applied functional MRI to study basal ganglia activation related to processing of temporal information. Subjects performed a time estimation and a frequency discrimination task on pairs of tones with stimulus ranges of approx. 1,000-1,400 ms and 1,000$1,015 \mathrm{~Hz}$, respectively. Frequency discrimination was used as a control condition on the understanding that it differs only in the (central) timing component. Although pitch separation might partially rely on temporal analysis in primary auditory cortex function (Griffiths et al. 1998), such processes depend on the particular sensory modality, and can thus be assumed to be independent from potential central timekeeper(s). On the basis of the patterns of brain activation, we shall discuss potential correlations between single components of models of temporal cognition (Gibbon et al. 1997; Nichelli 1993) and their potential neural substrates.

The analysis of the imaging data has two major aspects: the timing-related activation of the putamen (as compared to frequency discrimination) and the overall pattern of activity during time estimation (as compared to rest), which illustrates the more complex network of specific and unspecific components. The activation cluster in the right putamen, as displayed in Fig. 2, shows a characteristic profile, which would be expected for a process specific to the timing component. Having controlled for non-timing related aspects with the active control task, this supports our hypothesis of the basal ganglia, and more specifically the putamen, as a central structure in temporal information processing. This is in line with many behavioural and pharmacological studies on time processing and dopaminergic function (Gibbon et al. 1997). Our findings are an additional fMRI demon- 
stration of involvement of the putamen in perceptual timing and extend previous studies in several respects. In contrast to some previous studies, we have used a closely matched active control task to control for aspects like discrimination, attention, or decision-making. Furthermore, an adaptive algorithm was applied to adjust interindividual variability of performance to a set criterion level (see also Holcomb et al. 1998).

So far, studies on basal ganglia involvement in timing have focused on motor timing rather than on perceptual timing, such as in tapping experiments (Rao et al. 1997). An initial fMRI study of Hinton et al. (1996) on a smaller population showed basal ganglia activity in a timing task (peak-interval procedure), but with less control for attentional and memory effects. Maquet et al. (1996) detected increased regional cerebral blood flow (rCBF) using PET in a temporal generalisation task involving visual stimuli, but found no significant difference from a control task (intensity discrimination). In a later reassessment, however, the left putamen and a left cerebellar region were implicated in timing-relevant computations (Lejeune et al. 1997), but the question as to whether these really represent "basic time coding mechanisms" remains unresolved. In contrast to their finding, our timingspecific activation was located in the right, not left putamen. So far, it is not clear how and whether functional lateralisation is involved and which effects could contribute to it. As mentioned, the use of the left or right hand for pressing the response button was counterbalanced across subjects in our study, so confounding motor effects are unlikely. In another PET study, Jüptner et al. (1995) implicated cerebellar mechanisms to show timing-specific rCBF increase. They examined perceptual timing in a paired stimulus discrimination design similar to our approach, except that the control condition did not involve a discrimination challenge for the subjects. Therefore, attentional and memory demands differed markedly between the timing and the control task, influencing differential rCBF analysis. Nevertheless, their support for the cerebellum as a central mechanism is not necessarily in contradiction to our findings, as their PET scanning procedure was also spatially restricted, not covering the entire brain. It should be noted that the cerebellum was not completely covered in our study, and therefore we cannot reliably infer possible activations in this structure. Additionally, values of signal intensity for the cerebellum in our experiments appeared generally rather low on the images and therefore might have been eliminated partially by the conservative grey matter threshold given in the analysis software. Our data extend the finding of Jüptner and colleagues, assigning a more specific role of the basal ganglia in timing. In contrast to their study, our timing-related activation in the putamen is unlikely to be explained by differences in demands on attention and memory. Furthermore, the influence of individual performance differences was minimised through the use of the "weighted up-down method". We also eliminated influences of ongoing motor activity. Other factors, such as learning, are also insufficient to explain our result, as performance did not change significantly over the experiment.

The differential roles of the basal ganglia and the cerebellum in timing remain incompletely understood. Although it is now evident that there might be a role for both structures, it is unclear what the respective functions are. A separation along the temporal scale of stimuli (i.e. the hypothesis that the cerebellum supports timing in the millisecond range, and basal ganglia for seconds, respectively) is not in accordance with recent patient studies. For example, Parkinson's disease patients have been shown to be impaired in the millisecond range (Rammsayer and Classen 1997) and also in the second range (overview in Gibbon et al. 1997). Also, recent investigations show an impairment of cerebellar patients in the second range (Malapani et al. 1999), and not only for millisecond intervals. Our results are, however, in good accordance with the model of Gibbon and colleagues, who assume a central timing mechanism organised in the basal ganglia and attempt to explain the role of the cerebellum as a modulator of thalamocortical functioning. In the case of damage to the cerebellum, effects would be seen as increased variability, which is generally in accordance with previous studies (Gibbon et al. 1997). The question of shared mechanisms for motor and perceptual timing is still also a matter of discussion (Gibbon et al. 1997; Hazeltine et al. 1997).

Several methodological aspects need to be considered in the interpretation of these imaging findings, including our study. The main hypothesis to be tested in our experiment was the question of timing-specific regional brain activity, i.e. whether a neural substrate for a "central" timing mechanism would be detectable with fMRI. Our results demonstrate such timing-specific regional activity in the putamen, contrasting the timing to a control task carefully matched for unspecific components. More precisely, however, one could describe the resulting timing-specific neural correlate as an "explicit" timing mechanism. By "explicit" we refer to the property of this substrate to be involved (or challenged) when asking subjects to focus on the temporal aspect of stimuli, such as discriminating tones by their duration. However, in any control task using stimuli of equal length, subjects will still perceive the temporal aspect of identical duration, even if not focussing on it. At the core of many "internal clock" theories are independent oscillators, small networks giving a continuous repetitive signal, whose function would of course be ongoing and not necessarily modifiable by particular cognitive task demands. It therefore needs to be stressed that the putamen activation is not necessarily a direct correlate of the "internal clock". This aspect is particularly important in the design of functional imaging studies relying on subtractive designs. Functional MRI detects relative increases in signal intensity which are related to increases in neuronal function. As temporal information processing is a continuously ongoing process, the timingspecific activations would therefore need to be considered as a correlate of challenging timing mechanisms in the 
sense of modulating them and thereby increasing metabolic activity in underlying neural substrates. Such modulation (either pharmacologically or through increasing cognitive demands) might be detectable as increasing rates of firing, as shown in cellular recording studies in vitro, which claim to support the basal ganglia hypothesis of timing (Ruskin et al. 1999).

Beside the activation in the putamen, our second focus is on the more complex pattern demonstrated in the timing task vs rest condition. This allows us to relate the other modules of the information processing scheme (Nichelli 1993) to their potential neural substrates. Several regions have already been described in earlier studies. The superior temporal regions are known to be involved in the processing of auditory information and perceptual analysis of auditory information (Zatorre et al. 1994). For the further processing of such information contained in tones or trains of tones, the prefrontal cortex is essential. In particular, its dorsolateral aspect (the DLPFC) has been repeatedly demonstrated in tasks of working memory (D'Esposito et al. 2000; Owen 2000). In the case of tone pitch judgements, lateralisation to the right hemisphere has been described when the respective information is extracted from speech (Zatorre et al. 1992). Such right-side lateralisation for the DLPFC was also seen in our frequency task, as well as in time estimation. Given the somewhat divergent results of lesion studies and time estimation, differences in task demand or type have to be considered potentially leading to differences in prefrontal lateralisation. Previous neuroimaging studies have also demonstrated that activations of the DLPFC seem to be correlated with memory load (Braver et al. 1997; Rypma et al. 1999). Other studies on timing tasks have also found areas of the DLPFC, for example during the peak-interval procedure (Hinton et al. 1996), and have emphasised the role of prefrontal-striatal-thalamic loops for the maintenance of temporal information. These circuits might mediate the short-term storage of the intervals being compared. According to a model of middorsolateral and ventrolateral prefrontal function proposed by Petrides, the mid-dorsolateral part of the DLPFC (i.e. Brodmann's areas 46/9) is engaged in monitoring of information (Petrides 1995). This function is supposed to be organised irrespectively of the spatial or non-spatial nature of information being processed. According to this hypothesis, the ventrolateral prefrontal cortex, as seen in the activation of the right inferior frontal gyrus in our study, is important for selection, comparison, and decision processes in response to the task requirement. The lateralisation towards the right prefrontal cortex is also in accordance with neuropsychological studies that have suggested the right rather than left prefrontal cortex to be engaged in a frontoparietal network for timing functions (Harrington et al. 1998b).

Another prefrontal activation was located in the anterior cingulate/cingulate sulcus, a region implied in diverse computations underlying attentional control. Such activations are seen in a number of tasks, and their localisation and extent are dependent on the specific task demands (Paus et al. 1998). Cortical areas of the medial wall not only contribute to motor functions, but are also activated in cognitive tasks (Picard and Strick 2001). Only few data exist on an explicit role in timing, and these do not imply a specific role (Jackson et al. 1998). To some extent, the anterior cingulate activation as well as a part of the activations in basal ganglia (other than the timing-related right putamen region) are most likely effects of the motor component, i.e. response movements. Taken together, the activation of the DLPFC, the striatum and the thalamus together with the anterior cingulate can be well explained within the current models of temporal information processing, which emphasise the role of frontosubcortical circuits linking the prefrontal cortical areas with the striatum and thalamus (Gibbon et al. 1997).

Another region encountered in our analysis is the ventrolateral prefrontal/insular cortex region, which is potentially an interesting area for several reasons. Being mentioned in previous neuroimaging work on timing (Lejeune et al. 1997) as well as pitch processing (Zatorre et al. 1994), it might be essential for both tasks. As shown in our results, the activity is higher in time estimation than in frequency discrimination but lacking specificity for the timing task. Since this structure was not included in our anatomical hypothesis, our data only point to it in the sense of a trend, which needs further confirmation.

In conclusion, our study reveals two main findings. Time estimation elicits a widespread pattern of brain activity, which is very similar to a matched non-temporal active control task. However, activation differences in the putamen are apparent, supporting the notion of the striatum as a substrate for central timing mechanisms, or at least supporting a timing-specific function. Our data can be interpreted in the framework of current neuropsychological models of timing. It appears that the proposed "clock processes" might be associated with basal ganglia activity, whereas the memory and decision-making components rely on diverse cortical areas, such as the anterior cingulate and the DLPFC, in the context of frontothalamostriatal circuitry. Finally our results advocate the need for a reconsideration of the precise relation between the putamen and the cerebellum in timing. Conventional dichotomies no longer provide sufficient explanations for differential aspects and neurobiological models. In addition to behavioural studies and possibly also recording on the cellular level (e.g. Ruskin et al. 1999), functional neuroimaging might prove to be a major tool in this endeavour.

Acknowledgments This work was partially funded by the Bundesministerium für Bildung und Forschung (BMBF), grant 01ZZ9602. The authors would like to thank Mrs. I. Krumbein, B. Lange, K. Metzinger, and E. Wechsung from the Institute of Diagnostic and Interventional Radiology (Friedrich-Schiller University of Jena) for their excellent technical assistance with the fMRI experiments. 


\section{References}

Allan LG (1979) The perception of time. Percept Psychophys 26:340-354

Artieda J, Pastor MA, Lacruz F, Obeso JA (1992) Temporal discrimination is abnormal in Parkinson's disease. Brain 115:199-210

Braver TS, Cohen JD, Nystrom LE, Jonides J, Smith EE, Noll DC (1997) A parametric study of prefrontal cortex involvement in human working memory. Neuroimage 5:49-62

Brooks DJ, Ibanez V, Sawle GV, Quinn N, Lees AJ, Mathias CJ, Bannister R, Marsden CD, Frackowiak RS (1990) Differing patterns of striatal 18F-dopa uptake in Parkinson's disease, multiple system atrophy, and progressive supranuclear palsy. Ann Neurol 28:547-555

Damasceno BP (1996) Time perception as a complex functional system: neuropsychological approach. Int J Neurosci 85:237262

D'Esposito M, Postle BR, Rypma B (2000) Prefrontal cortical contributions to working memory: evidence from event-related fMRI studies. Exp Brain Res 133:3-11

Fraisse P (1957) Psychologie du temps. Presses Universitaires de France, Paris

Fraisse P (1963) The psychology of time. Harper and Row, New York

Friston KJ, Holmes AP, Worsley KJ, Poline JB, Frith CD, Frackowiak RSJ (1995) Statistical parametric maps in functional imaging: a general linear approach. Hum Brain Mapp 2:189-210

Frackowiak RSJ, Friston KJ, Frith CD, Dolan RJ, Mazziotta JC (1997) Human brain function. Academic Press, San Diego

Fuster JM (1995) Temporal processing. Ann N Y Acad Sci 769:173-181

Gibbon J, Allan L (1984) Timing and time perception. New York Academy of Sciences, New York

Gibbon J, Malapani C, Dale CL, Gallistel CR (1997) Toward a neurobiology of temporal cognition: advances and challenges. Curr Opin Neurobiol 7:170-184

Griffiths TD, Buchel C, Frackowiak RS, Patterson RD (1998) Analysis of temporal structure in sound by the human brain. Nat Neurosci 1:422-427

Häger F, Volz HP, Gaser C, Mentzel HJ, Kaiser WA, Sauer H (1998) Challenging the anterior attentional system with a continuous performance task: a functional magnetic resonance imaging approach. Eur Arch Psychiatry Clin Neurosci 248:161-170

Harrington DL, Haaland KY, Hermanowicz N (1998a) Temporal processing in the basal ganglia. Neuropsychology 12:3-12

Harrington DL, Haaland KY, Knight RT (1998b) Cortical networks underlying mechanisms of time perception. J Neurosci 18:1085-1095

Hazeltine E, Helmuth LL, Ivry RB (1997) Neural mechanisms of timing. Trends Cogn Sci 1:163-169

Hellström A, Lang H, Portin R, Rinne J (1997) Tone duration discrimination in Parkinson's disease. Neuropsychologia 35:737-740

Hinton SC, Meck WH, McFall JR (1996) Peak-interval timing in humans activates fronto-striatal loops. Neuroimage 3:224

Holcomb HH, Medoff DR, Caudill PJ, Zhao Z, Lahti AC, Dannals RF, Tamminga CA (1998) Cerebral blood flow relationships associated with a difficult tone recognition task in trained normal volunteers. Cereb Cortex 8:534-542

Holmes AP, Friston KJ (1998) Generalisability, random effects and population inference. Neuroimage 7:S754

Ivry RB (1996) The representation of temporal information in perception and motor control. Curr Opin Neurobiol 6:851-857

Ivry RB, Keele SW (1989) Timing functions of the cerebellum. J Cogn Neurosci 1:136-152

Jackson PA, Kesner RP, Amann K (1998) Memory for duration: role of hippocampus and medial prefrontal cortex. Neurobiol Learn Mem 70:328-348
Jüptner M, Rijntjes M, Weiller C, Faiss JH, Timmann D, Mueller SP, Diener HC (1995) Localization of a cerebellar timing process using PET. Neurology 45:1540-1545

Kaernbach C (1991) Simple adaptive testing with the weighted updown method. Percept Psychophys 49:227-229

Lejeune H, Maquet P, Bonnet M, Casini L, Ferrara A, Macar F, Pouthas V, Timsit-Berthier M, Vidal F (1997) The basic pattern of activation in motor and sensory temporal tasks: positron emission tomography data. Neurosci Lett 235:21-24

Malapani C, Rakitin B, Levy R, Meck WH, Deweer B, Dubois B, Gibbon J (1998) Coupled temporal memories in Parkinson's disease: a dopamine-related dysfunction. J Cogn Neurosci 10:316-331

Mangels JA, Ivry RB, Shimizu N (1998) Dissociable contributions of the prefrontal and neocerebellar cortex to time perception. Brain Res Cogn Brain Res 7:15-39

Maquet P, Lejeune H, Pouthas V, Bonnet M, Casini L, Macar F, Timsit-Berthier M, Vidal F, Ferrara A, Degueldre C, Quaglia L, Delfiore G, Luxen A, Woods R, Mazziotta JC, Comar D (1996) Brain activation induced by estimation of duration: a PET study. Neuroimage 3:119-126

Meck WH (1996) Neuropharmacology of timing and time perception. Brain Res Cogn Brain Res 3:227-242

Nichelli P (1993) The neuropsychology of human temporal information processing. In: Boller F, Grafman J (eds) Handbook of neuropsychology, vol 8. Elsevier, Amsterdam, pp 339371

Nichelli P, Venneri A, Molinari M, Tavani F, Grafman J (1993) Precision and accuracy of subjective time estimation in different memory disorders. Brain Res Cogn Brain Res 1:87-93

Oldfield RC (1971) The assessment and analysis of handedness: the Edinburgh inventory. Neuropsychologia 9:97-113

Owen AM (2000) The role of the lateral frontal cortex in mnemonic processes: the contribution of functional neuroimaging. Exp Brain Res 133:33-43

Pastor MA, Artieda J, Jahanshahi M, Obeso JA (1992) Time estimation and reproduction is abnormal in Parkinson's disease. Brain 115:211-225

Paus T, Koski L, Caramanos Z, Westbury C (1998) Regional differences in the effects of task difficulty and motor output on blood flow response in the human anterior cingulate cortex: a review of 107 PET activation studies. Neuroreport 9:R37-R47

Penhune VB, Zatorre RJ, Evans AC (1998) Cerebellar contributions to motor timing: a PET study of auditory and visual rhythm reproduction. J Cogn Neurosci 10:752-765

Petrides M (1995) Functional organization of the human frontal cortex for mnemonic processing. Ann N Y Acad Sci 769:85-96

Picard N, Strick PL (2001) Imaging the premotor areas. Curr Opin Neurobiol 11:663-672

Rammsayer T (1992) An experimental comparison of the weighted up-down method and the transformed up-down method. Bull Psychonomic Soc 30:425-427

Rammsayer TH (1997a) Effects of body core temperature and brain dopamine activity on timing processes in humans. Biol Psychol 46:169-192

Rammsayer TH (1997b) Are there dissociable roles of the mesostriatal and mesolimbocortical dopamine systems on temporal information processing in humans? Neuropsychobiology 35:36-45

Rammsayer T, Classen W (1997) Impaired temporal discrimination in Parkinson's disease: temporal processing of brief durations as an indicator of degeneration of dopaminergic neurons in the basal ganglia. Int J Neurosci 91:45-55

Rao SM, Harrington DL, Haaland KY, Bobholz JA, Cox RW, Binder JR (1997) Distributed neural systems underlying the timing of movements. J Neurosci 17:5528-5535

Ruskin DN, Bergstrom DA, Kaneoke Y, Patel BN, Twery MJ, Walters JR (1999) Multisecond oscillations in firing rate in the basal ganglia: robust modulation by dopamine receptor activation and anesthesia. J Neurophysiol 81:2046-2055 
Rypma B, Prabhakaran V, Desmond JE, Glover GH, Gabrieli JD (1999) Load-dependent roles of frontal brain regions in the maintenance of working memory. Neuroimage 9:216-226

Talairach J, Tournoux P (1988) Co-planar stereotaxic atlas of the human brain. Thieme, Stuttgart

Volz HP, Gaser C, Häger F, Rzanny R, Mentzel HJ, KreitschmannAndermahr I, Kaiser WA, Sauer H (1997) Brain activation during cognitive stimulation with the Wisconsin Card Sorting Test - a functional MRI study on healthy volunteers and schizophrenics. Psychiatry Res 75:145-157
Wetherill GB, Chen H, Vasudeva RB (1966) Sequential estimation of quantal response curves: a new method of estimation. Biometrika 53:439-454

Zatorre RJ, Evans AC, Meyer E, Gjedde A (1992) Lateralization of phonetic and pitch discrimination in speech processing. Science 256:846-849

Zatorre RJ, Evans AC, Meyer E (1994) Neural mechanisms underlying melodic perception and memory for pitch. J Neurosci 14:1908-1919 